\title{
Differentiation of human amniotic epithelial cells into Schwann-like cells via indirect co-culture with Schwann cells in vitro
}

\author{
SHUANG ZHU ${ }^{1 *}$, JIACHUN LI $^{1 *}$, QINGTANG ZHU ${ }^{1}$, TING DAI $^{2}$, BO HE ${ }^{1}$, \\ XIANG ZHOU ${ }^{1}$, JIANPING XIANG ${ }^{1}$ and XIAOLIN LIU ${ }^{1}$ \\ ${ }^{1}$ Department of Microsurgery and Hand Surgery, \\ The First Affiliated Hospital of Sun Yat-sen University; ${ }^{2}$ Department of Biotechnology, \\ School of Basic Science, Guangzhou Medical University, \\ Guangzhou, Guangdong 510080, P.R. China
}

Received December 19, 2013; Accepted September 24, 2014

DOI: $10.3892 / \mathrm{mmr} .2014 .2881$

\begin{abstract}
Human amniotic epithelial cells (hAECs) exhibit multi-lineage differentiation ability. The present study investigated the possibility that hAECs possess the potential to differentiate into Schwann-like cells using an in vitro indirect co-culture approach. hAECs were isolated via enzymatic digestion, and immunocytochemistry and flow cytometry were performed to identify the hAECs. The hAECs were co-cultured with Schwann cells (SCs) to differentiate the hAECs into Schwann-like cells via induced proximity. The expression of typical S-100 SC markers in the co-cultured hAECs was determined via immunocytochemistry. For the functional experiments, reverse transcription quantitative polymerase chain reaction (RT-qPCR) was performed to measure the expression levels of nerve growth factor (NGF), brain-derived neurotrophic factor (BDNF) and glial cell-derived neurotrophic factor (GDNF) mRNA. In addition, neurite outgrowth was measured in PC12 cells following co-culture with the differentiated hAECs. Subsequent to co-culture with SCs for 21 days, the hAECs exhibited spindle-like morphology. The immunocytochemistry results revealed that the co-cultured hAECs expressed S-100, indicating differentiation into Schwann-like cells. RT-qPCR revealed that NGF, BDNF and GDNF expression was upregulated upon differentiation. The average axon length of the PC12 cells increased from
\end{abstract}

Correspondence to: Professor Jianping Xiang or Professor Xiaolin Liu, Department of Microsurgery and Hand Surgery, The First Affiliated Hospital of Sun Yat-sen University, 58 Zhongshan 2nd Road, Guangzhou, Guangdong 510080, P.R. China

E-mail: 328438430@qq.com; gzjianpingxiang@qq.com

E-mail: gzxiaolinliu@hotmail.com

*Contributed equally

Key words: human amniotic epithelial cells, co-culture, differentiation, PC12 cells, Schwann cells
$21.32 \pm 5.45$ to $51.32 \pm 8.56 \mu \mathrm{m}$ subsequent to co-culture with the differentiated hAECs. These results demonstrate that this indirect co-culture microenvironment induced the hAECs to differentiate into Schwann-like cells that exhibited the morphological, phenotypic and functional characteristics of SCs. Therefore, the use of differentiated hAECs that exhibit the characteristics of SCs provides a promising alternative to the present techniques used for peripheral nerve regeneration.

\section{Introduction}

Annually, $>3,500,000$ patients worldwide suffer from peripheral nerve injuries, accounting for $3 \%$ of all trauma patients. Among the various types of peripheral nerve injury, transection injuries, in which the nerve trunk is completely separated, particularly those that result in large defects, may produce a marked impact on patients' quality of life, and in these cases, reconstructive surgery is required as a therapeutic strategy to achieve nerve regeneration. Several studies have attempted to accelerate the regeneration of traumatized nerves via the application of microsurgical techniques (1-6). However, despite advancements in these techniques, complete recovery is rarely achieved $(7,8)$.

Schwann cells (SCs) are considered to be the primary structural and functional cells of the peripheral nervous system, and these cells exert a critical supportive role in peripheral nerve regeneration $(9,10)$. The transplantation of cultured SCs into various peripheral nerve grafts scaffolds has been demonstrated to promote axonal regeneration across nerve gaps (11). However, the clinical application of SCs remains controversial as obtaining sufficiently large numbers of SCs is difficult. In the past few decades, bone marrow stromal cells (BMSCs) and adipose-derived stem cells (ADSCs) have been considered as alternative cell sources for tissue regeneration following peripheral nerve injury, as these cells are capable of selectively differentiating into SCs and enhancing axonal regeneration $(12,13)$. Each of these cell types has advantages; however, numerous problems have been associated with the use of each of the currently available cell sources, including 
complications regarding cell survival, immune rejection, tumorigenesis and ethical issues. Therefore, the development of an ideal cell source is important to address these problems. Recently, human amniotic epithelial cells (hAECs) have been reported to exhibit stem cell characteristics, and these cells have been shown to differentiate into liver cells, fat cells, heart cells and nerve cells in vitro (14-19). hAECs are easily accessible and highly abundant since the amnion is normally discarded following birth. Furthermore, hAECs exhibit low immunogenicity and have the potential to differentiate into various types of functional cell (20). These characteristics render hAECs an alternative source of adult stem cells for SC transplantation for peripheral nerve regeneration.

In the present study, whether hAECs possess the potential to differentiate into Schwann-like cells was investigated via indirect co-culture of hAECs with SCs in vitro. Immunocytochemical staining was performed to determine the biological features of the differentiated cells. Co-culture of the differentiated hAECs with PC12 cells was conducted to assess the effect of differentiated hAECs on axon regeneration.

\section{Materials and methods}

Isolation and culture of hAECs. In brief, discarded placentas from uncomplicated cesarean resections at 37-40 weeks gestational age were obtained from The First Affiliated Hospital of Sun Yat-sen University (Guangzhou, China) with the approval of the University of Sun Yat-sen Institutional Review Board. The amnion layer was mechanically peeled away from the chorion and washed multiple times using phosphate-buffered saline (PBS; Invitrogen, Carlsbad, CA, USA) to remove the blood. The amnion layer was then cut into $2 \times 2 \mathrm{~cm}$ sections and processed according to previously described methods (21). To release the hAECs, the amnion sections were incubated at $37^{\circ} \mathrm{C}$ in $0.05 \%$ trypsin containing $0.2 \mathrm{~g} / 1$ ethylenediaminetetraacetic acid (Invitrogen/Gibco-BRL; Carlsbad, CA, USA). Subsequently, the trypsin was inactivated by adding $10 \mathrm{ml}$ Dulbecco's modified Eagle's medium/F-12 Ham nutrient mixture (DMEM/F-12; 1:1; Gibco-BRL) supplemented with $10 \%$ heat-inactivated fetal bovine serum (FBS; Invitrogen Life Technologies,). The cells from the initial $10 \mathrm{~min}$ of digestion were discarded to remove any debris. The cells from the second and third 30-min digestions were pooled and washed three times with PBS. The hAECs were then plated on six-well dishes at a seeding density of $20,000 \mathrm{cells} / \mathrm{cm}^{2}$ in DMEM/F-12 (1:1) culture medium supplemented with $10 \%$ FBS, $1 \%$ penicillin/streptomycin (Invitrogen), $1 \%$ GlutaMAX $^{\mathrm{TM}}$ (Invitrogen) and $10 \mathrm{ng} / \mathrm{ml}$ epidermal growth factor (EGF; PeproTech, Rocky Hill, NJ, USA). The hAECs from the initial culture were subcultured to passage 1 using a split ratio of 1:3. The above-mentioned culture medium was replaced every three days.

Isolation and culture of SCs. Rat SCs were harvested as previously described, with minor modifications (22). Briefly, for collection of the sciatic nerves, the male Sprague-Dawley rats (1-3 days old, weighing 8-15 g; Laboratory Animal Center of Sun Yat-sen University, Guangzhou, China) were anesthetized by cooling on ice one at a time. Once the animal had stopped moving it was decapitated, and the head placed into a collection bag. The body was spayed with $70 \%$ ethanol. The sciatic nerves were exposed, dissected out under a binocular microscope (Leica S6E,Wetzlar, Germany) and placed in a sterile culture dish (3-cm diameter; Costar, Corning Inc., Corning, NY, USA) containing ice-cold PBS with $1 \%$ penicillin/streptomycin, where they were maintained until the nerves had been harvested from all of the rat. Then, the sciatic nerves were enzymatically dissociated via sequential incubation at $37^{\circ} \mathrm{C}$ in $0.3 \%$ collagenase type II (Sigma-Aldrich, St. Louis, Missouri, USA) and $0.125 \%$ trypsin (Invitrogen/ Gibco-BRL) for 30 and $10 \mathrm{~min}$, respectively. The mixture was triturated, centrifuged and resuspended in DMEM/F-12 supplemented with $10 \% \mathrm{FBS}$ and $1 \%$ penicillin/streptomycin. On the following day, $10 \mu \mathrm{M}$ cytosine arabinoside (Sigma-Aldrich) was added, and the mixture was allowed to incubate for an additional $48 \mathrm{~h}$ to remove any fibroblasts. Subsequently, the culture was maintained in DMEM/F-12 supplemented with $10 \% \mathrm{FBS}, 1 \%$ penicillin/streptomycin and $2 \mathrm{ng} / \mathrm{ml}$ heregulin (Sigma-Aldrich, St. Louis, MO, USA) to stimulate SC proliferation. All media and supplements were purchased from Invitrogen/Gibco-BRL. All experiments were performed in accordance with the guidelines for the use of experimental animals from the National Institutes of Health and ethical approval for this study was obtained from the Ethics Committee of Sun Yat-sen University.

Viability and proliferation assays for hAECs. The hAECs were harvested during the logarithmic growth phase and digested using trypsin. To examine cell proliferation and viability, the hAECs $\left(5 \times 10^{3}\right.$ cells/well) were plated in 96-well dishes and incubated in DMEM/F-12 supplemented with $10 \%$ FBS. Plates were incubated at $37^{\circ} \mathrm{C}$ in $5 \% \mathrm{CO}_{2}$ for 24 or 48 hours. Four hours prior to the end of the incubation time, $20 \mu \mathrm{l}$ 3-(4, 5-dimethylthiazol-2-yl) 2,5diphenyltetrazolium bromide solution (MTT, Sigma-Aldrich) was added to each well. Following a $4 \mathrm{~h}$ of incubation, plates were centrifuged and culture medium was removed. Precipitated formazan crystals were dissolved by adding $150 \mu \mathrm{L}$ DMSO to each well. The microplates were shaken at room temperature for $10 \mathrm{~min}$. A microplate reader (OPTImax; Molecular Dynamics, Sunnyvale, CA, USA) was used to measure the absorbance (A) of each well at $540 \mathrm{~nm}$, and the average values were obtained. Experiments were repeated $\geq 3$ times, and the data are presented as the means \pm standard deviation (SD).

Flow cytometric analysis. Isolated hAECs were harvested and washed using $0.5 \%$ bovine serum albumin (BSA)/0.01 M PBS solution. Following fixation using 4\% paraformaldehyde (Invitrogen), the cells were resuspended in $0.5 \% \mathrm{BSA} / \mathrm{PBS}$ solution and incubated for $1 \mathrm{~h}$ at room temperature with fluorescein isothiocyanate (FITC)-conjugated primary mouse monoclonal antibody against CD34 (MA1-12103; Thermo Fisher Scientific, Waltham, MA, USA; 1:200). Negative controls were produced using mouse immunoglobulin G 1-isotyped antibodies conjugated to FITC (Beckman Coulter, Marseille, France). The samples were analyzed using a BD FACSCanto ${ }^{\mathrm{TM}}$ II system (BD Biosciences, San Jose, CA, USA). A total of $3 \times 10^{4}$ events were captured using the BD FACSDiva ${ }^{\mathrm{TM}}$ software (BD Biosciences) histogram feature. The data were further analyzed using CellQuest software (BD Biosciences) 
and WinMDI software version 2.9 (Scripps Research Institute; La Jolla, CA, USA).

Differentiation of hAECs via co-culture with SCs. hAECs were co-cultured with SCs in a double-chamber system with SCs on the upper level and hAECs on the lower level of the culture plate. The cultures were suspended in DMEM/F-12 culture medium at a density of $2 \times 10^{4}$ cells/well in a 6-well dish (Costar; Corning Inc., Corning, NY, USA). Culture inserts (Costar; Corning Inc.), each containing $8 \times 10^{4} \mathrm{SCs}$, were placed in four of the six wells using one insert/well. An insert membrane with a pore size of $0.4 \mu \mathrm{m}$ diameter was used to permit only the transmission of soluble factors and to prevent direct interaction between the hAECs and the SCs. Half the volume of the co-culture medium was replaced every three days. hAECs under identical conditions but without co-culture with SCs served as negative controls and SCs alone served as positive controls.

Immunocytochemistry. The characteristics of differentiated hAECs at the protein expression level were assessed via immunocytochemistry. The cells were fixed at room temperature with 4\% paraformaldehyde for $10 \mathrm{~min}$. Subsequently, the cells were washed with PBS, followed by permeabilization for 30 min using $0.1 \%$ Triton X-100. Non-specific binding sites were blocked by incubation with 5\% goat serum for $1 \mathrm{~h}$. The following primary antibodies were employed for immunocytochemistry: monoclonal mouse against Pan-cytokeratin (4545; $1: 200)$ and monoclonal mouse against S-100 (5529; 1:200) obtained from Cell Signaling Technology (Danvers, MA, USA). The cells were incubated in the primary antibodies overnight at $4^{\circ} \mathrm{C}$. The cells were washed three times with PBS and incubated with the corresponding goat secondary antibodies $(4408 ; 1: 500)$ for $2 \mathrm{~h}$ at room temperature. Subsequent to washing twice with PBS, the cells were incubated in 4',6'-diamidino-2-phenylindole dihydrochloride $(1: 1,000$; Sigma-Aldrich) for $5 \mathrm{~min}$ at room temperature to counterstain the cell nuclei. The specimens were washed three times with PBS, each for $5 \mathrm{~min}$, and were then mounted using an antifade solution. Immunolabeled cells were examined via fluorescence microscopy (Zeiss Axiovision Z1, Oberkochen, Germany). Images were digitally recorded and processed using Image-Pro Plus (Media Cybernetics, Rockville, MD, USA).

RNA isolation. To collect total RNA, $2 \times 10^{6}$ cells were seeded on $10 \mathrm{~cm}$ diameter dishes in $8 \mathrm{ml}$ growth medium. The cultures were maintained at $37^{\circ} \mathrm{C}$ in a humidified atmosphere of $5 \% \mathrm{CO}_{2} / 95 \%$ air. The media were replaced, including the drug of interest. After six days, total RNA was isolated using an Isogen kit (Nippon Gene, Tokyo, Japan) according to the manufacturer's instructions. RNA quantity and purity were determined via spectrophotometry (DU-65; Beckman Coulter, Fullerton, CA, USA).

Reverse trancription quantitative polymerase chain reaction $(R T-q P C R)$. RT-qPCR was performed using an RNA PCR kit [avian myeloblastosis virus (AMV)] version 2.1 (Takara Bio, Inc., Shiga, Japan) according to the manufacturer's instructions and with a PCR Thermal Cycler (Takara Bio, Inc.). First-strand cDNA was synthesized from total RNA (1 mg) using AMV reverse transcriptase XL primed using 50 pmol random 9 mers (Takara Bio, Inc.). The first-strand reaction was conducted as follows: $30^{\circ} \mathrm{C}$ for $10 \mathrm{~min}, 50^{\circ} \mathrm{C}$ for $30 \mathrm{~min}, 99^{\circ} \mathrm{C}$ for $5 \mathrm{~min}$ and $58^{\circ} \mathrm{C}$ for $5 \mathrm{~min}$. The reverse-transcription reaction products $(10 \mathrm{ml})$ were utilized as templates for PCR using $0.2 \mathrm{mM}$ of each primer. Primers specific to rat nerve growth factor (NGF), glial cell-derived neurotrophic factor (GDNF), brain-derived neurotrophic factor (BDNF) and glyceraldehyde 3-phosphate dehydrogenase (GAPDH) were designed using Primer Express 2.0 software (Applied Biosystems, Foster City, CA, USA) and were obtained from Shanghai HuiRui Biotechnology Co., Ltd. (Shanghai, China). The PCR products were separated via precast $2 \%$ agarose gel (Daiichi Pure Chemicals Co., Ltd., Tokyo, Japan) electrophoresis and visualized via SYBR-Green 2 (FMC Bioproducts, Rockland, ME, USA) staining using a ultraviolet trans-illuminator (UVP Inc., San Gabriel, CA, USA). The signal intensity of the PCR products was determined using Image $\mathbf{J}$ software. The expression levels of the GDNF, BDNF and NGF PCR products were quantified as determined by the intensity of the PCR products compared with that of the corresponding GAPDH reactions.

Functional co-culture assay with PC12 cells. PC12 cells were plated in six-well chamber slides pre-coated with collagen (Sigma-Aldrich) at a density of 1,000 cells/well. The PC12 cells were incubated for $24 \mathrm{~h}$ in DMEM supplemented with $5 \%$ horse serum, $10 \% \mathrm{FBS}, 100 \mathrm{U} / \mathrm{ml}$ penicillin and $100 \mu \mathrm{g} / \mathrm{ml}$ streptomycin. At $24 \mathrm{~h}$ prior to plating the PC12 cells, 293T cells and hAECs differentiated towards a SC phenotype were seeded on $0.4 \mu \mathrm{m}$ pore size culture inserts at a seeding density of $2 \times 10^{4}$ cells/insert and incubated at $37^{\circ} \mathrm{C}, 95 \%$ humidity and $5 \% \mathrm{CO}_{2}$. After $48 \mathrm{~h}$, the inserts were checked for cell adherence and were added to the PC12 chamber slides such that molecules secreted by the cells were able to permeate to the PC12 cells without direct cell contact. Neuron-like outgrowth was measured using an Olympus IX81 microscope (Olympus Corporation, Tokyo, Japan) and a U-CMAD 3 camera (Olympus Corporation). Cell body size and axon length were analyzed using SIS image analysis software version 3.2 (Olympus Soft Imaging Solutions GmbH, Münster, Germany). PC12 cells with axons longer than the average cell diameter were used for data analysis. Cell axon length was analyzed using image-pro-plus (SIS,Münster, Germany).

Statistical analysis. All data were analyzed using SPSS 11.0 statistical software (SPSS, Inc., Chicago, IL, USA). The experimental data are expressed as the mean \pm SD. The Student's t-test was performed to compare the values. $\mathrm{P}<0.05$ was considered to indicate a statistically significant difference.

\section{Results}

Identification of hAECs. The viability of the isolated cells was $>95 \%$, as determined via trypan blue staining. In the presence of EGF, hAECs proliferated robustly and formed a confluent monolayer of cobblestone-shaped epithelial cells (Fig. 1A). Approximately all isolated cells reacted with antibodies against pan-cytokeratin, which revealed the epithelial nature of the cells and the lack of contamination with human amniotic mesenchymal stromal cells (Fig. 1B). The absence of 


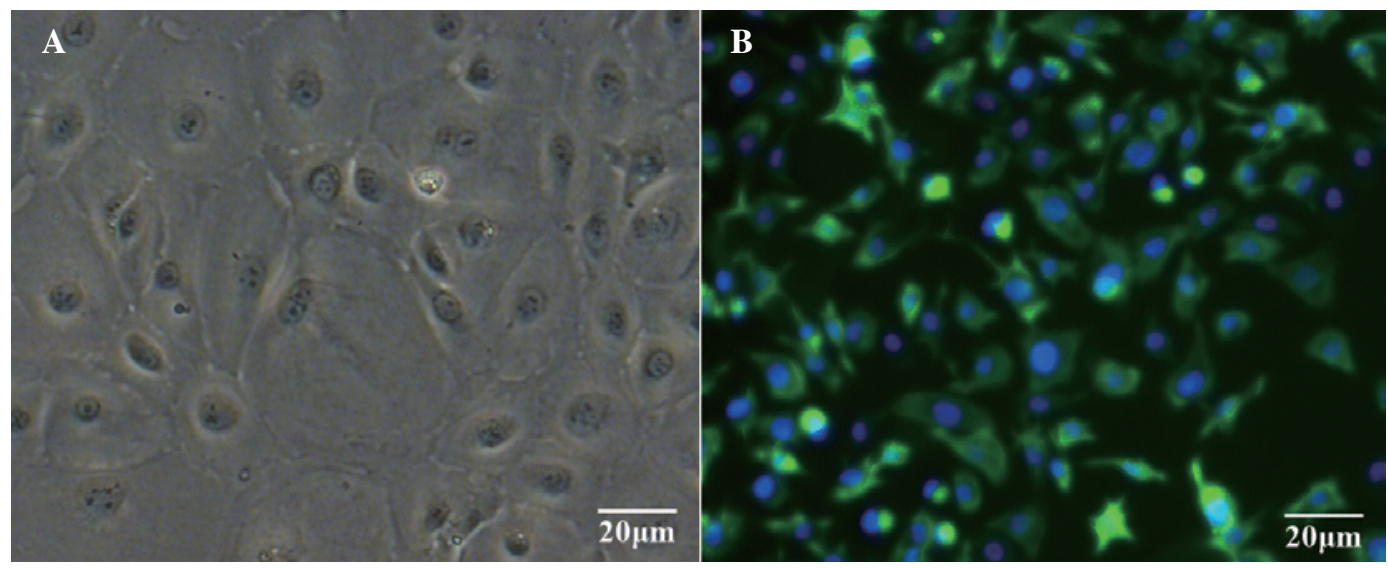

Figure 1. Identification of human amniotic epithelial cells. (A) Microscopic examination of human amniotic epithelial cells seven days after culture (magnification, x40). (B) Immunostaining against pan-cytokeratin (magnification, x20).

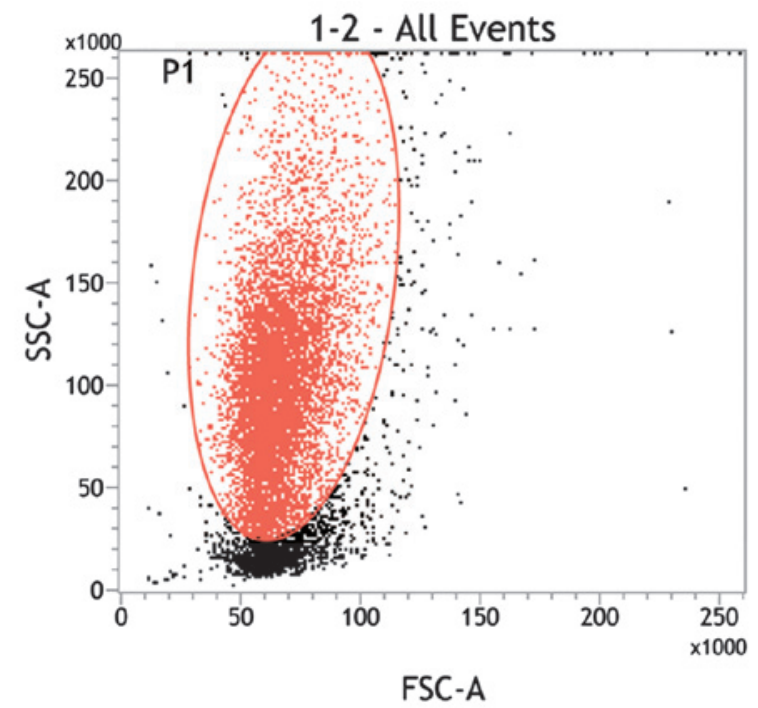

$1-2-P 1$

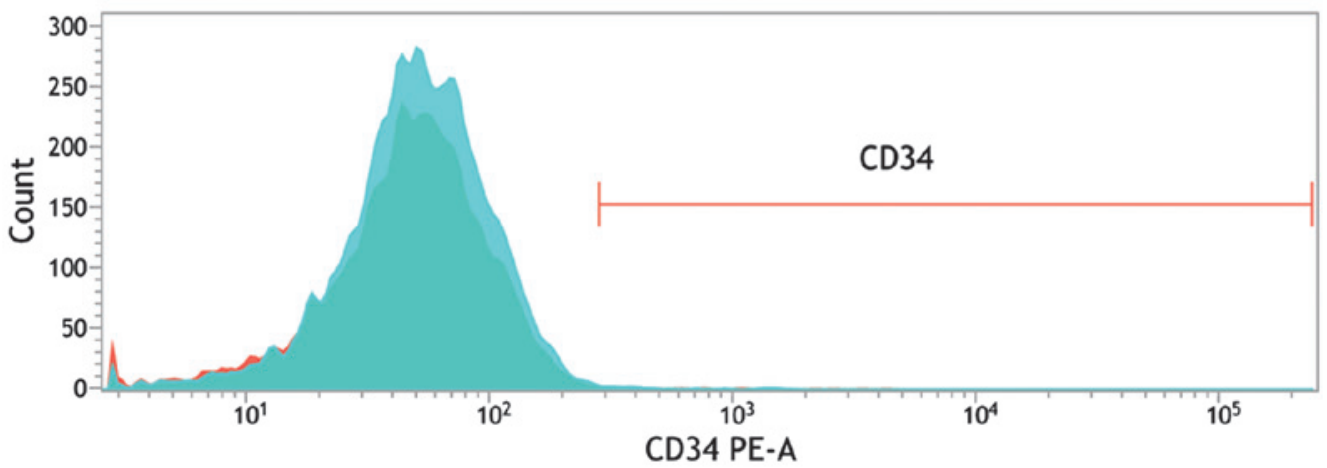

Figure 2. Flow cytometry analysis of human amniotic epithelial cells. SSC, side light scatter; FSC, forward light scatter; CD, cluster of differentiation; PE, phycoerythrin.

CD34-positive cells in this population indicates that the isolates were not contaminated with hematopoietic stem cells, such as umbilical cord blood cells or embryonic fibroblasts (Fig. 2).

Identification of differentiated hAECs. hAECs cultured alone appeared as a monolayer of cobblestone-shaped epithelial cells (Fig. 3A). Following co-culture of the cells with SCs for
21 days, certain hAECs exhibited a typical bipolar or tripolar morphology (Fig. 3B). As compared with undifferentiated hAECs (Fig. 3C), differentiated hAECs exhibited positive expression of S-100 (Fig. 3D).

Expression levels of NGF, BDNF and GDNF mRNA following differentiation. To examine the biological function 

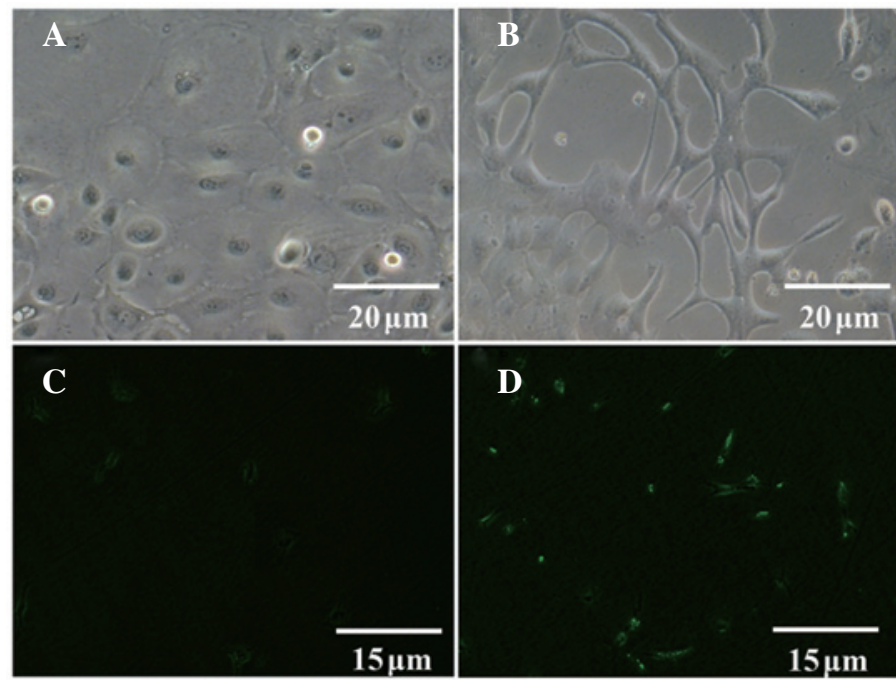

\section{$\mathbf{D}$}
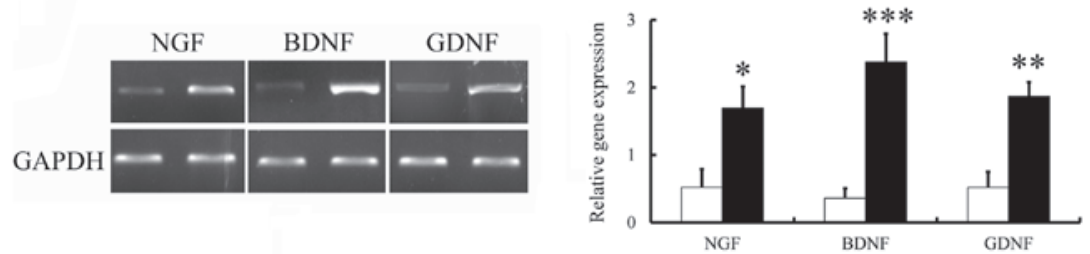

Figure 4. mRNA expression levels of neurotrophic factors in hAECs. The results of reverse transcription quantitative polymerase chain reaction analysis of NGF, BDNF and GDNF mRNA expression levels. Representative results are shown, and the data are presented as the mean \pm standard deviation (n=6). A two-tailed Mann-Whitney U test was performed for statistical analysis of differential expression levels. ${ }^{*} \mathrm{P}<0.05,{ }^{* * *} \mathrm{P}<0.01$ and ${ }^{* * *} \mathrm{P}<0.001$. hAECs, human amniotic epithelial cells; NGF, nerve growth factor; BDNF, brain-derived neurotrophic factor; GDNF, glial cell-derived neurotrophic factor.
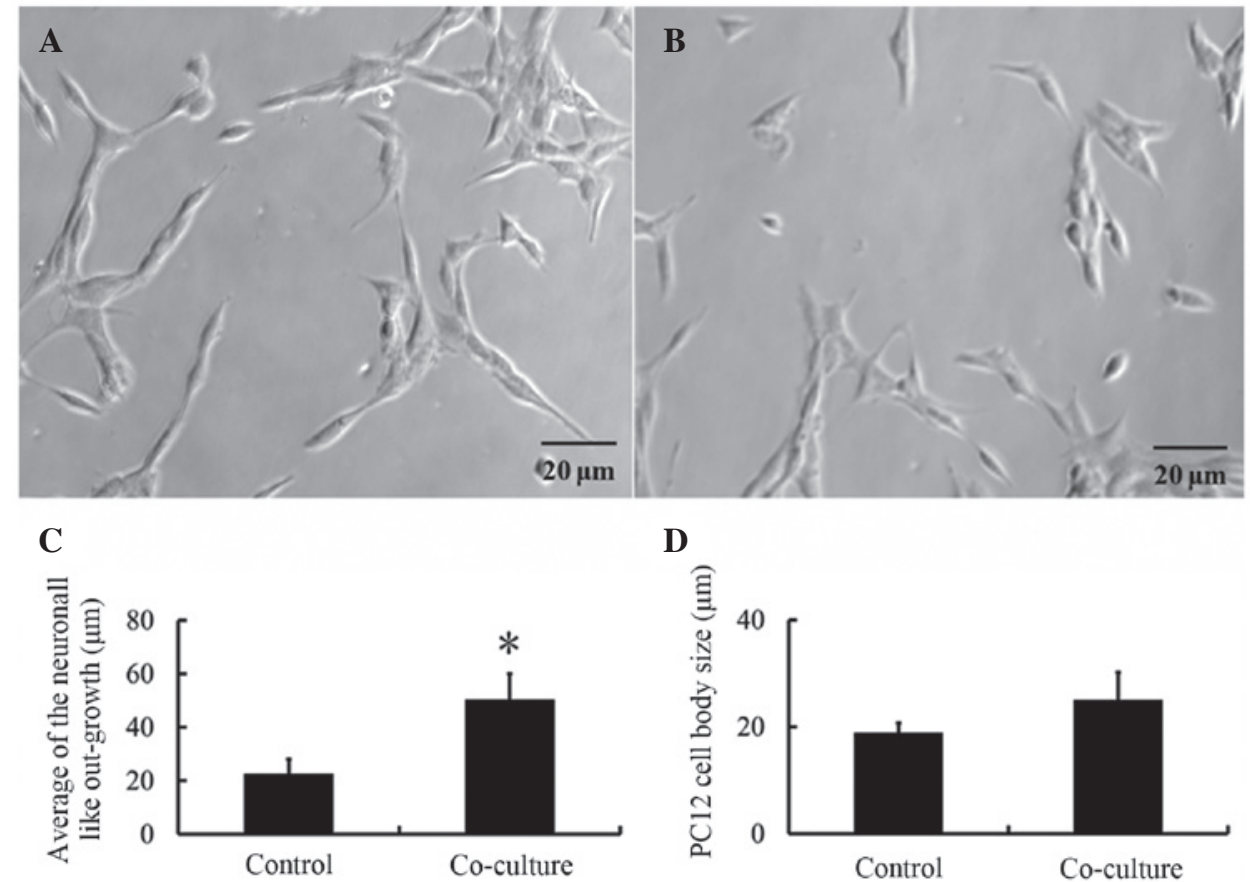

Figure 5. Effect of co-culture with differentiated hAECs on the axonal sprouting of PC12 cells. Microscopic images of (A) PC12 cells co-cultured with 293T cells as a control or (B) with differentiated hAECs. Comparison of (C) the average axon length of PC12 cells and (D) the average diameter of the PC12 cell bodies subsequent to co-culture for 10 days. The data are presented as the means \pm standard deviation of PC12 cell cultures in media ( $\mathrm{n}=6$ ). A two-tailed Mann-Whitney U test was performed for statistical analysis. ${ }^{*} \mathrm{P}<0.05$ compared with the control group. hAECs, human amniotic epithelial cells. 
of the differentiated cells, the expression levels of the NGF, BDNF and GDNF neurotrophic factors in the cells were measured via RT-qPCR using specific primers, as described above. Neurotrophic factors are polypeptides or proteins that are released by neurons or non-neuronal cells that exert marked effects on the central and peripheral nervous systems (23). Exogenous administration of NGF has been reported to completely prevent the death of axotomized sensory neurons $(24,25)$. Subsequent to co-culture of the hAECs with SCs, the expression levels of NGF mRNA in the hAECs increased 1.55-fold as compared with treatment with saline (Fig. 4). Subsequently, whether co-culture results in a sustained increase in BDNF and GDNF mRNA expression levels was determined. The BDNF and GDNF mRNA levels were also significantly increased following co-culture (1.5-fold, $\mathrm{P}<0.05$; and 1.8-fold, $\mathrm{P}<0.05$, respectively).

PC12 cell morphology. To determine the effect of differentiated hAECs on PC12 cell axon regeneration subsequent to co-culture of PC12 cells with differentiated hAECs, the number of PC12 cells that exhibited axonogenesis, the cell body sizes and the overall length of the outgrown fibers were quantified. Fig. 5 clearly presents the biological activity of the differentiated cells. Statistical analysis of the neuron-like outgrowth of the PC12 cells revealed significant induction of axonogenesis following co-culture $(\mathrm{P}<0.05 ; 51.32 \pm 8.56 \mu \mathrm{m})$ as compared with the control group $(21.32 \pm 5.45 \mu \mathrm{m})$. In addition, the average cell body size of the PC12 cells was determined during the culture period since alterations in cell morphology and size indicate higher metabolic activity induced by external stimuli. The cell body sizes were measured for each group. The average PC12 cell body size increased following incubation in the co-culture. However, these marginal changes were not statistically significant.

\section{Discussion}

In the present study, hAECs were differentiated into SCs using a co-culture method. The results revealed that, following differentiation, the hAECs exhibited a spindle-like morphology. The hAECs were immunopositive for the S-100 SC markers. Subsequently, the function of the differentiated hAECs was analyzed. The RT-qPCR results revealed that NGF, BDNF and GDNF expression was upregulated as compared with the control group. In addition, following the co-culture of differentiated hAECs with PC12 cells, the differentiated cells significantly increased the average axon length of the PC12 cells.

In recent years, numerous strategies have been developed to prepare nerve conduits involving biomaterials that incorporate growth factors, cells and natural microarchitectural structures $(26,27)$. To more effectively repair nerve defects, the construction of nerve grafts that integrate these factors is a promising alternative to the employment of autologous nerves. SCs are a good candidate for use as seed cells. However, treatment with primary SCs as a cell therapeutic remains limited by the associated donor site morbidity (28). In addition, for clinical applications, the time period required to generate a sufficient quantity of cells is not always feasible (29). Therefore, various studies have attempted to identify an alternative method. hAECs, which exhibit characteristics of embryonic and pluripotent stem cells, may be utilized for cell therapy without legal or ethical problems (30). The differentiation of hAECs has been widely investigated. Using $\beta$-mercaptoethanol, Banerjee et al (31) found that human amniotic membranes were differentiated towards the SC lineage in vitro. Following differentiation, the cells expressed BDNF and GDNF, a finding concurrent with that of the present study.

Contrary to BMSCs or ADSCs, hAECs are not stem cells. However, hAECs do possess the multi-lineage differentiation potential required to develop into tissues of three germ layers (ectodermal, mesodermal and endodermal lineages), a finding which has been demonstrated by multiple studies (32-38). In addition, the demonstration of hAEC expression of pluripotent and multipotent markers, such as octamer-binding protein (Oct)-3/4, SRY-related HMG-box 2, Nanog, fibroblast growth factor 4 , reduced expression 1 , frizzled class receptor 9 , bone marrow stromal cell antigen 1 and ATP-binding cassette sub-family $\mathrm{G}$ member 2, provides further support for the multi-lineage differentiation potential of hAECs $(32,39,40)$.

In the present study, the overall proportion of differentiated hAECs remained low. Two factors may account for this phenomenon. On one hand, the quantity of co-cultured hAECs was large. Incubation of an excessive number of cells together may not only restrict cell growth and differentiation but also limit the relative effect of the proteins secreted by the SCs. Conversely, Miki et al (32) found that hAECs grow as three layers when cultured in dishes: A layer of cells in the supernatant, an intermediate layer and a layer of cells attached to the culture dish. Furthermore, the expression levels of the pluripotent markers Oct-4 and Nanog were elevated in the cells that were present above the basal layer of hAECs, which indicates that the cells above the basal layer exhibited greater differentiation ability. This phenomenon may partially explain why particular hAECs differentiate more quickly and easily. In the present study, due to the only partial differentiation of hAECs into SCs observed, the function of the cells following co-culture was subsequently examined.

PC12 cells are a well-defined and widely used model for the analysis of axon regeneration (41). In the present study, following co-culturing, the average axon length was observed to increase from $21.32 \pm 5.45$ to $51.32 \pm 8.56 \mu \mathrm{m}$. The PC12 cell body size may also have increased, as alterations in cell morphology and size indicated a higher metabolic activity induced by external stimuli. A marginal, but not significant, increase in the average cell body size was demonstrated. This result implied that unlike average axon length, cell body size may not provide an appropriate marker of regeneration.

In conclusion, in general, using a co-culture technique, hAECs were demonstrated in the present study to differentiate towards SCs in vitro. The differentiated hAECs exhibited certain characteristics of SCs and greatly increased the axon length of PC12 cells. These promising results indicate that this method may provide a good strategy for the treatment of nerve injuries in humans.

\section{Acknowledgements}

This study was supported by grants from Guangdong Medical Science and Technology Research Fund (grant 
no. A2011163), Doctoral Startup Project of Guangdong Natural Science Foundation (grant no. S201204006336), the National High Technology Research and Development Program of China (863 Program, grant no. 012AA020507), the Nature Science Grant of Guangdong China (grant no. 9251008901000017, and 2012AA020507), the National Basic Research Program of China (973 Program, grant no. 2014CB542201) and the 985 program of Sun Yat-sen University (grant no. 90035-3283312).

\section{References}

1. He B, Zhu Q, Chai Y, et al: Safety and efficacy evaluation of a human acellular nerve graft as a digital nerve scaffold: a prospective, multicentre controlled clinical trial. J Tissue Eng Regen Med: Feb 25, 2013 (Epub ahead of print).

2. Okui N, Yamamoto M,Fukuhira Y, Kaneko H, Hirata H: A new nerve coaptation technique using a biodegradable honeycombpatterned film. Microsurg 32: 466-474,2012.

3. Meng H1, Li M, You F, Du J and Luo Z: Assessment of processed human amniotic membrane as a protective barrier in rat model of sciatic nerve injury. Neurosci Lett 496:48-53,2011.

4. Wangensteen KJ and Kalliainen LK: Collagen tube conduits in peripheral nerve repair: a retrospective analysis. Hand (N Y) 5: 273-277, 2010.

5. Kehoe S, Zhang XF and Boyd D.: FDA approved guidance conduits and wraps for peripheral nerve injury: a review of materials and efficacy. Injury 43: 553-572,2012.

6. Elliot D, Lloyd M, Hazari A, Sauerland S and Anand P.: Relief of the pain of neuromas-in-continuity and scarred median and ulnar nerves in the distal forearm and wrist by neurolysis, wrapping in vascularized forearm fascial flaps and adjunctive procedures. J Hand Surg Eur Vol 35: 575-582, 2010.

7. Kline DG and Hudson AR: Vertebral artery compression. J Neurosurg 83: 759, 1995.

8. Schmidt CE and Leach JB: Neural tissue engineering: Strategies for repair and regeneration. Annu Rev Biomed Eng 5: 293-347, 2003.

9. He B, Liu SQ, Chen Q, et al: Carboxymethylated chitosan stimulates proliferation of Schwann cells in vitro via the activation of the ERK and Akt signaling pathways. Eur J Pharmacol 667: 195-201, 2011.

10. Yazdani SO, Golestaneh AF, Shafiee A, et al: Effects of low level laser therapy on proliferation and neurotrophic factor gene expression of human schwann cells in vitro. J Photochem Photobiol B 107: 9-13, 2012.

11. Gambarotta G, Fregnan F, Gnavi S and Perroteau I: Neuregulin 1 role in Schwann cell regulation and potential applications to promote peripheral nerve regeneration. Int Rev Neurobiol 108: 223-256, 2013.

12. Hu J, Zhu QT, Liu XL, Xu YB and Zhu JK: Repair of extended peripheral nerve lesions in rhesus monkeys using acellular allogenic nerve grafts implanted with autologous mesenchymal stem cells. Exp Neurol 204: 658-666, 2007.

13. Sowa Y, Imura T, Numajiri T, Nishino $\mathrm{K}$ and Fushiki S: Adipose-derived stem cells produce factors enhancing peripheral nerve regeneration: Influence of age and anatomic site of origin. Stem Cells Dev 21: 1852-1862, 2012.

14. Manuelpillai U, Tchongue J, Lourensz D, et al: Transplantation of human amnion epithelial cells reduces hepatic fibrosis in immunocompetent $\mathrm{CCl}_{4}$-treated mice. Cell Transplant 19: 1157-1168, 2010.

15. Miki T, Marongiu F, Dorko K, Ellis EC and Strom SC: Isolation of amniotic epithelial stem cells. Curr Protoc Stem Cell Biol 1: 1E.3, 2010 .

16. Murphy S, Rosli S, Acharya R, et al: Amnion epithelial cell isolation and characterization for clinical use. Curr Protoc Stem Cell Biol 1: 1E.6, 2010.

17. Parolini O, Alviano F, Bagnara GP, et al: Concise review: Isolation and characterization of cells from human term placenta: Outcome of the first international Workshop on Placenta Derived Stem Cells. Stem Cells 26: 300-311, 2008.
18. Tsutsumi S, Shimazu A, Miyazaki K, et al: Retention of multilineage differentiation potential of mesenchymal cells during proliferation in response to FGF. Biochem Biophys Res Commun 288: 413-419, 2001

19. Toda A, Okabe M, Yoshida T and Nikaido T: The potential of amniotic membrane/amnion-derived cells for regeneration of various tissues. J Pharmacol Sci 105: 215-228, 2007.

20. Akle CA, Adinolfi M, Welsh KI, Leibowitz S and McColl I: Immunogenicity of human amniotic epithelial cells after transplantation into volunteers. Lancet 2: 1003-1005, 1981.

21. Fatimah SS, Ng SL, Chua KH, et al: Value of human amniotic epithelial cells in tissue engineering for cornea. Hum Cell 23: 141-151, 2010.

22. Weinstein DE and Wu R: Isolation and purification of primary Schwann cells. Curr Protoc Neurosci 3: 3.17, 2001.

23. Baloh RH, Enomoto H, Johnson EJ, Milbrandt J: The GDNF family ligands and receptors - implications for neural development. Curr Opin Neurobiol 10:103-110,2000

24. Rich KM, Yip HK, Osborne PA, Schmidt RE and Johnson EJ Jr: Role of nerve growth factor in the adult dorsal root ganglia neuron and its response to injury. J Comp Neurol 230: 110-118, 1984.

25. Taniuchi M, Clark HB and Johnson EJ: Induction of nerve growth factor receptor in Schwann cells after axotomy. Proc Natl Acad Sci USA 83: 4094-4098, 1986.

26. Zhang L, Lv X, Tong X, Jia H and Li Z: Study on molecular mechanism for improving neural regeneration after repair of sciatic nerve defect in rat by acellular nerve allograft. Synapse 66 : 52-60, 2012.

27. Zheng C, Zhu Q, Liu X, et al: Effect of platelet-rich plasma (PRP) concentration on proliferation, neurotrophic function and migration of Schwann cells in vitro. J Tissue Eng Regen Med: May 31, 2013 (Epub ahead of print).

28. Xu Y, Liu L, Li Y, et al: Myelin-forming ability of Schwann cell-like cells induced from rat adipose-derived stem cells in vitro. Brain Res 1239: 49-55, 2008.

29. Kingham PJ, Kalbermatten DF, Mahay D, et al: Adipose-derived stem cells differentiate into a Schwann cell phenotype and promote neurite outgrowth in vitro. Exp Neurol 207: 267-274, 2007.

30. Xue S, Chen C, Dong W, et al: Therapeutic effects of human amniotic epithelial cell transplantation on double-transgenic mice co-expressing APPswe and PS1 $\Delta$ E9-deleted genes. Sci China Life Sci 55: 132-140, 2012.

31. Banerjee A, Nürnberger S, Hennerbichler S, et al: In toto differentiation of human amniotic membrane towards the Schwann cell lineage. Cell Tissue Bank 15: 227-239, 2014.

32. Miki T, Lehmann T, Cai H, Stolz DB and Strom SC: Stem cell characteristics of amniotic epithelial cells. Stem Cells 23: $1549-1559,2005$

33. Ilancheran S, Michalska A, Peh G, et al: Stem cells derived from human fetal membranes display multilineage differentiation potential. Biol Reprod 77: 577-588, 2007.

34. Hou Y, Huang Q, Liu T and Guo L: Human amnion epithelial cells can be induced to differentiate into functional insulin-producing cells. Acta Biochim Biophys Sin (Shanghai) 40: 830-839, 2008.

35. Miki T and Strom SC: Amnion-derived pluripotent/multipotent stem cells. Stem Cell Rev 2: 133-142, 2006.

36. Elwan MA and Sakuragawa N: Evidence for synthesis and release of catecholamines by human amniotic epithelial cells. Neuroreport 8: 3435-3438, 1997.

37. Wei JP, Zhang TS, Kawa S, et al: Human amnion-isolated cells normalize blood glucose in streptozotocin-induced diabetic mice. Cell Transplant 12: 545-552, 2003.

38. Sakuragawa N, Enosawa S, Ishii T, et al: Human amniotic epithelial cells are promising transgene carriers for allogeneic cell transplantation into liver. J Hum Genet 45: 171-176, 2000.

39. Simat SF, Chua KH, Abdul Rahman H, Tan AE and Tan GC: The stemness gene expression of cultured human amniotic epithelial cells in serial passages. Med J Malaysia 63 (Suppl A): 53-54, 2008.

40. Tan GC, Simat SF, Abdul Rahman H, Tan AE and Chua KH: Quantitative RT PCR approach to evaluate the neurogenic and gliagenic gene expression of cultured human amniotic epithelial cells. Med J Malaysia 63 (Suppl A): 51-52, 2008.

41. Zhou X, He X, He B, et al: Etifoxine promotes glial-derived neurotrophic factor-induced neurite outgrowth in PC12 cells. Mol Med Rep 8: 75-80, 2013 